\title{
Individualizing Treatment for Patients With Schizoaffective Disorder
}

\author{
Eduard Vieta, MD, PhD \\ Bipolar Disorder Program and the Clinical Institute of Neuroscience, Hospital Clinic, \\ University of Barcelona, IDIBAPS, CIBERSAM, Barcelona, Spain
}

Although compelling diagnostic definitions and evidence-based treatment recommendations for schizoaffective disorder are lacking, clinicians can still develop an effective, individualized treatment regimen for patients with this condition. The steps necessary to help patients with schizoaffective disorder reach and maintain remission are to confirm the diagnosis, evaluate the patient's predictors of outcome, be aware of the available pharmacotherapeutic options and prescribe appropriate medications, and implement psychotherapy once patients achieve remission.

\section{Confirm the Diagnosis}

Because schizoaffective disorder encompasses symptoms of mood disorders as well as psychosis, clinicians should complete a differential diagnosis to rule out bipolar disorder and schizophrenia. Patients may have either bipolar or depressive subtypes of schizoaffective disorder, which are defined as having predominately manic or depressive symptoms, respectively, in addition to psychosis. ${ }^{1}$ For more information on confirming a diagnosis of schizoaffective disorder, see the J Clin Psychlopedia activity "Differentially Diagnosing Schizoaffective Disorder."

\section{Evaluate the Predictors of Outcome}

After confirming a diagnosis of schizoaffective disorder, clinicians should evaluate the patient's predictors of outcome, which include previous levels of functioning, number of prior episodes, nature of psychotic symptoms, and extent of cognitive impairment. Initially assessing these features is critical in determining an effective, individualized treatment plan and anticipating the patient's course of illness and outcome.

Previous functioning. Patients with schizoaffective disorder tend to have levels of social and occupational functioning that are better than those with schizophrenia but worse than those with bipolar disorder. ${ }^{2}$ Using a cluster analysis, Lipkovich et $\mathrm{al}^{3}$ determined that the better the social and occupational functioning that patients with schizoaffective disorder have at baseline, the more likely they will have good treatment outcomes.

Number of episodes. As patients experience more episodes of schizoaffective disorder, their chances of achieving full recovery dramatically 
decline. For example, Marneros et $\mathrm{al}^{4}$ found that the primary factors in persisting psychosocial deficits among patients with schizoaffective disorder included having a high number of episodes and a high annual frequency of episodes. Further, having a high number of episodes also influenced the development of negative social consequences due to the illness.
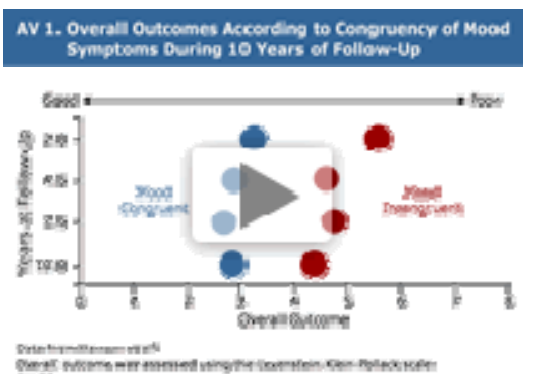

AV 1. Overall Outcomes According to Congruency of Mood Symptoms During 10 Years of Follow-Up (00:20)

Nature of psychosis. By definition, patients with schizoaffective disorder have psychotic symptoms, but the persistence and presentation of those symptoms can predict outcomes for patients. For example, patients with less persistent psychotic symptoms have a better prognosis than those with more persistent psychosis. ${ }^{4}$ And, patients with mood-incongruent psychotic symptoms, ie, inappropriate to the mood episode, are likely to have poor outcomes ( $A \vee 1$ ), including poor psychosocial functioning as well as the presence of major psychotic symptoms. ${ }^{5}$

Cognitive impairment. Because cognitive impairment is closely related to functioning, clinicians should evaluate deficits in patients with schizoaffective disorder. The premorbid intellectual, language, and behavioral functioning of patients with schizoaffective disorder was found to be similar to that of schizophrenia patients and worse than that of bipolar disorder patients. ${ }^{6}$ Additionally, one study ${ }^{7}$ found that neurocognitive performance was a better predictor of occupational functioning, ie, employment status and the ability to obtain a job, than were the clinical symptoms of schizoaffective disorder.

Implement Pharmacotherapy

After evaluating each patient's predictors of outcome, formulate an individualized treatment plan based on the available evidence, which is categorized below by medication in order of the studies' methodological strength. The amount of data for schizoaffective disorder is limited because most trials have examined subsamples of schizoaffective disorder in addition to schizophrenia. Very limited data is available for compounds not listed below, including conventional antipsychotics, lithium, or valproate. 


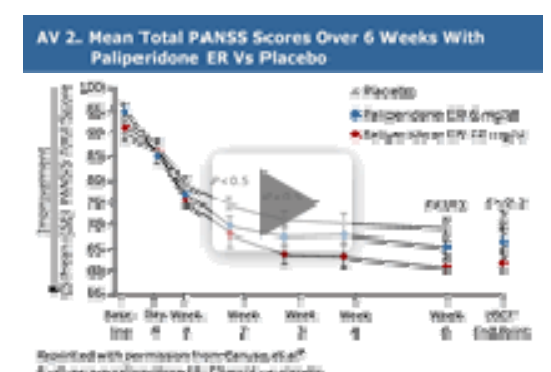

AV 2. Mean Total PANSS Scores Over 6 Weeks With Paliperidone ER Vs Placebo $(00: 21)$

Paliperidone. This is the only compound that has been assessed in a specifically designed, large, randomized, placebo-controlled study ${ }^{8}$ for this condition. Canuso et $\mathrm{al}^{8}$ examined acute schizoaffective disorder $(\mathrm{N}=316)$ and found that the atypical antipsychotic paliperidone ER $(12 \mathrm{mg} / \mathrm{d})$ significantly improved total scores on the PANSS (AV 2, P=.003), the CGI-SSCA $(P<.001)$, the YMRS (for those with prominent manic symptoms, $\mathrm{P}<.001$ ), and the HDRS (for those with prominent depressive symptoms, $\mathrm{P}<.05)$ compared with placebo. Overall, the medication was well tolerated; headache and tremor were the most common adverse events, although headache was reported more often in the placebo group.

Ziprasidone and aripiprazole. Pooled data from 2 double-blind placebocontrolled trials ${ }^{9}$ of schizophrenia, focusing on the subset of patients with schizoaffective disorder $(n=115)$ reported significant dose-related improvements with ziprasidone (with $160 \mathrm{mg} / \mathrm{d}$ having the largest effect) on the BPRS total score, BPRS Core Items subscale, BPRS Manic Items, and the CGI-S $(\mathrm{P}<.01$ for all) compared with placebo. The most common medicationinduced adverse events were general pain and headache, but a notable lack of many adverse events, such as EPS and sexual dysfunction, was apparent.

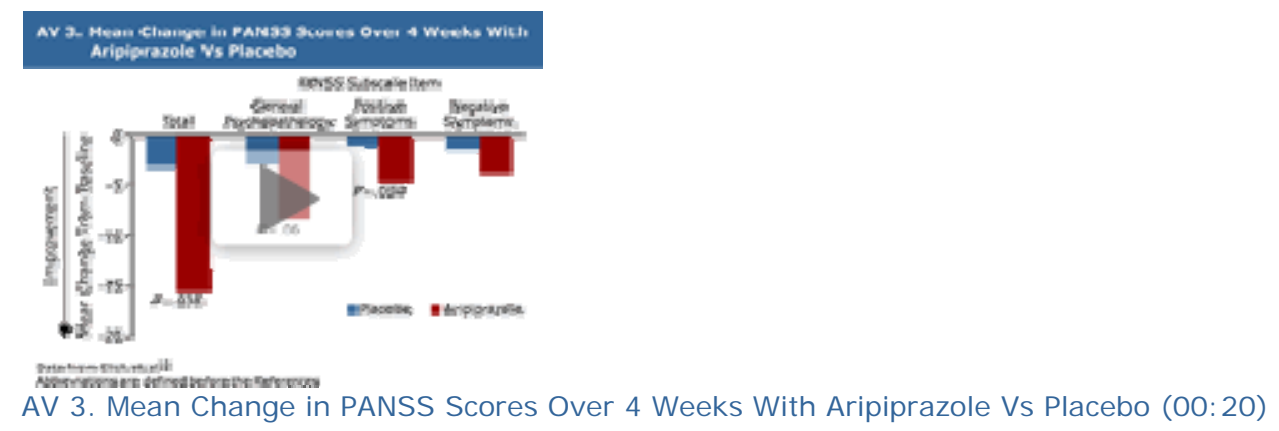

Another pooled analysis ${ }^{10}$ of 2 double-blind placebo-controlled trials of a schizoaffective disorder subset of subjects $(n=179)$ found that aripiprazole (up to $30 \mathrm{mg} / \mathrm{d}$ ) significantly improved total scores on the PANSS $(P=.038$ ) and the CGI-I $(P=.023)$ compared with placebo (AV 3). Commonly reported adverse effects of aripiprazole were headache, agitation, and insomnia, although the placebo group reported higher rates for each of these events. 
Topiramate. A small placebo-controlled, double-blind pilot study ${ }^{11}$ of topiramate (mean dose $=276 \mathrm{mg} / \mathrm{d}$ ) added to mood stabilizers or atypical antipsychotics for schizoaffective disorder, bipolar type $(\mathrm{N}=48)$ did not show efficacy. Similar reductions in PANSS, YMRS, and CGI-S scores were reported for both topiramate and placebo. No serious adverse events were found, although the topiramate group experienced significantly more weight loss $(\mathrm{P}<.02)$ than placebo-treated patients.

Olanzapine. A comparative double-blind study ${ }^{12}$ of olanzapine (mean dose $=11.5 \mathrm{mg} / \mathrm{d}$ ) versus haloperidol (mean dose $=10 \mathrm{mg} / \mathrm{d}$ ) in a schizoaffective disorder subset $(n=300)$ demonstrated significantly greater improvements in total scores for the PANSS $(P=.003)$, the BPRS $(P=.002)$, and the MADRS $(P<.001)$ with olanzapine. Patients treated with olanzapine had significantly less occurrence of all adverse events, except for increased appetite, than haloperidol.

Risperidone. In a double-blind comparative study ${ }^{13}$ of risperidone (mean dose $=5.5 \mathrm{mg} / \mathrm{d}$ ) and haloperidol (mean dose $=10.8 \mathrm{mg} / \mathrm{d}$ ), patients with schizoaffective disorder $(\mathrm{N}=62)$ had symptomatic improvement with both medications on the PANSS, the CARS-M, and the HDRS, but the differences were not significant. Risperidone was better tolerated than haloperidol, with significantly more haloperidol-treated patients experiencing EPS ( $P=.03)$. In an open study ${ }^{14}$ of schizoaffective disorder or bipolar disorder $(N=541)$, the addition of risperidone ( mean dose $=3.9 \mathrm{mg} / \mathrm{d}$ ) to mood stabilizers showed significant improvement on the YMRS, the HDRS, the PANSS, and the CGI $(P<.0001$ for all). The adjunctive risperidone was well tolerated, with only $2.4 \%$ of subjects experiencing weight gain, which was the most commonly reported adverse event.

Treatment adherence. To ensure that patients have the opportunity to achieve remission, clinicians should educate patients about their illness to help them have awareness and insight, address any concerns patients have about taking medication, and identify potential safety problems and barriers to adherence, such as co-occurring personality or substance use disorders. For patients who have difficulty adhering to a daily medication regimen, clinicians may consider implementing a long-acting injectable formulation of the medication. Additionally, psychotherapy can be used, but should only be employed when patients have reached remission. For more information on improving treatment adherence, see the J Clin Psychlopedia activity "I mproving Treatment Adherence in Schizoaffective Disorder." 


\section{Add Psychoeducation}

When patients have reached remission, which is the time when they are best suited to understand, learn, and develop strategies to cope with the illness, psychoeducation can help to improve patient outcomes by increasing illness insight and awareness, improving treatment adherence, and helping patients and caregivers identify early signs of relapse. Although no studies specific to schizoaffective disorder are available, data from bipolar disorder and schizophrenia trials may be helpful.

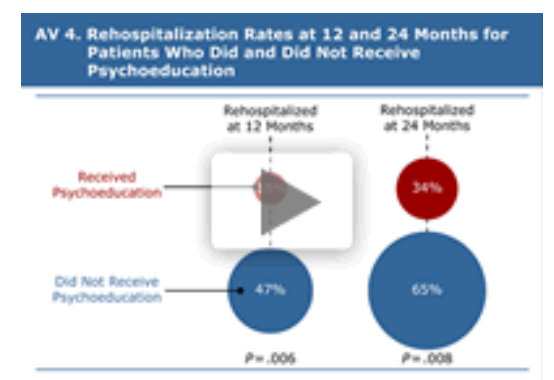

AV 4. Rehospital Psychoeducation (00:25)

In studies of schizophrenia, psychoeducation improved patients' knowledge of their illness, ${ }^{15,16}$ treatment adherence, ${ }^{17}$ and quality of life ${ }^{15}$ as well as reduced rehospitalization rates for those with 2 to 5 previous psychotic episodes (AV 4). ${ }^{17}$

In a study ${ }^{18}$ of bipolar disorder, group psychoeducation reduced the number of relapses and recurrences, reduced the number and length of hospitalizations, and increased the time to any mood recurrence.

Additionally, caregiver psychoeducation has been shown to reduce the recurrence and increase the time to intervention for any mood episode. ${ }^{19}$ Individualize Treatment

Individualizing treatment for patients with schizoaffective disorder requires merging the evidence base from schizoaffective disorder, schizophrenia, and bipolar disorder and tailoring treatment plans to each patient's specific schizoaffective subtype, eg, bipolar type or depressive type.

Bipolar type. Atypical antipsychotics should be given for patients with schizoaffective disorder, bipolar type, particularly when psychotic and manic symptoms are present. Evidence in bipolar disorder suggests that combining an atypical antipsychotic and mood stabilizer is the preferred first-line treatment ${ }^{20}$; both specific medication choices should be guided by individual symptom profiles and treatment tolerability. After the patient reaches 
remission, psychoeducation should be instituted and the medication regimen that helped the patient achieve remission should be continued through the maintenance phase; atypical antipsychotic monotherapy is also acceptable during this phase of treatment.

Depressive type. Evidence for the depressive subtype of schizoaffective disorder is so limited that the recommendations here are from clinical practice rather than clinical trials. Generally, using an atypical antipsychotic plus an antidepressant or a mood stabilizer should be sufficient to manage schizoaffective disorder, depressive type, but again, the individual patient's symptomatic profile and tolerability should guide specific medication choices. Additionally, psychoeducation should be implemented once the patient achieves remission and enters the maintenance phase of treatment.

\section{For Clinical Use}

- Complete a differential diagnosis of schizoaffective disorder, distinguishing it from schizophrenia and bipolar disorder

- Evaluate each patient's predictors of outcome, ie, previous levels of functioning, number of prior episodes, nature of psychotic symptoms, and extent of cognitive impairment

- Be aware of the pharmacotherapeutic options available for schizoaffective disorder and their level of evidence

- Allow individual patient's symptomatic profile and tolerability to guide specific medication selection

- Implement psychoeducation after the patient has reached remission

\section{Complete the differential diagnosis training module and then come back to complete the posttest.}

\section{Drug Names}

aripiprazole (Abilify), haloperidol (Haldol and others), lithium (Eskalith and others), olanzapine (Zyprexa), paliperidone (Invega), risperidone (Risperdal and others), topiramate (Topamax and others), ziprasidone (Geodon)

\section{Abbreviations}

BPRS $=$ Brief Psychiatric Rating Scale, CARS-M = Clinician-Administered Rating Scale for Mania, CGI-I/S/SCA = Clinical Global ImpressionsImprovement/Severity/Schizoaffective Disorder scale, EPS = extrapyramidal symptoms, ER = extended release, HDRS = Hamilton Depression Rating Scale, MADRS = Montgomery-Asberg Depression Rating Scale, PANSS = Positive and Negative Syndrome Scale, YMRS = Young Mania Rating Scale 

Edition, Text Revision. Washington, DC: American Psychiatric Association; 2000.

2. Benabarre A, Vieta E, Colom F, et al. Bipolar disorder, schizoaffective disorder and schizophrenia: epidemiologic, clinical and prognostic differences. Eur Psychiatry. 2001;16(3): 167- 172.

3.

Lipkovich IA, Deberdt W, Csernansky JG, et al. Defining "good" and "poor" outcomes in patients with schizophrenia or schizoaffective disorder: a multidimensional data-driven approach. Psychiatry Res. 2009; 170(2-3): 161-167.

4. Marneros A, Rohde A, Deister A. Factors influencing the long-term outcome of schizoaffective disorders. Psychopathology. 1993;26(2-3):215-224.

5. Harrow M, Grossman LS, Herbener ES, et al. Ten-year outcome: patients with schizoaffective disorders, schizophrenia, affective disorders and mood-incongruent psychotic symptoms. $\mathrm{Br}$ J Psychiatry. 2000; 177(5): 421-426.

6. Reichenberg A, Weiser M, Rabinowitz J, et al. A population-based cohort study of premorbid intellectual, language, and behavioral functioning in patients with schizophrenia, schizoaffective disorder, and nonpsychotic bipolar disorder. Am J Psychiatry. 2002; 159(12): 2027-2035.

7. Kaneda Y, Jayathilak K, Meltzer HY. Determinants of work outcome in schizophrenia and schizoaffective disorder: role of cognitive function. Psychiatry Res. 2009; 169(2): 178-179.

8. Canuso CM, Lindenmayer JP, Kosik-Gonzalez C, et al. A randomized, double-blind, placebocontrolled study of 2 dose ranges of paliperidone extended-release in the treatment of subjects with schizoaffective disorder. J Clin Psychiatry. 2010; 71(5):587-598.

9. Keck PE, Reeves KR, Harrigan EP, et al. Ziprasidone in the short-term treatment of patients with schizoaffective disorder: results from two double-blind, placebo-controlled, multicenter studies. J Clin Psychopharmacol. 2001;21(1):27-35.

10. Glick ID, Mankoski R, Eudicone JM, et al. The efficacy, safety, and tolerability of aripiprazole for the treatment of schizoaffective disorder: results from a pooled analysis of a sub-population of subjects from two randomized, double-blind, placebo-controlled, pivotal trials. J Affect Disord. 2009; 115(1-2): 18-26.

11. Chengappa KNR, Kupfer DJ, Parepally H, et al. A placebo-controlled, random-assignment, parallel-group pilot study of adjunctive topiramate for patients with schizoaffective disorder, bipolar type. Bipolar Disorders. 2007;9(6):609-617.

12. Tran PV, Tollefson GD, Sanger TM, et al. Olanzapine versus haloperidol in the treatment of schizoaffective disorder: acute and long-term therapy. Br J Psychiatry. 1999; 174(1): 15-22.

13. Janicak PG, Keck PE Jr, Davis JM. A double-blind, randomized, prospective evaluation of the efficacy and safety of risperidone versus haloperidol in the treatment of schizoaffective disorder. J Clin Psychopharmacology. 2001;21(4):360-368.

14. Vieta E, Goikolea JM, Corbella B, et al. Risperidone safety and efficacy in the treatment of bipolar and schizoaffective disorders: results from a 6-month, multicenter, open study. J Clin Psychiatry. 2001;62(10):818-825.

15. Sibitz I, Amering M, Gössler R, et al. One-year outcome of low-intensity booster sessions versus care as usual in psychosis patients after short-term psychoeducational intervention. Eur Psychiatry. 2007;22(4):203-210.

16. Vreeland B, Minsky S, Yanon PT, et al. Efficacy of the team solutions program for educating patients about illness management and treatment. Psychiatr Serv. 2006;57(6):822-828.

17. Pitschel-Walz G, Bäuml J, Bender W, et al. Psychoeducation and compliance in the treatment of schizophrenia: results of the Munich Psychosis Information Project Study. J Clin Psychiatry. 2006;67(3): 443-452.

18. Colom F, Vieta E, Martinez-Arán A, et al. A randomized trial on the efficacy of group psychoeducation in the prophylaxis of recurrences in bipolar disorder whose disease is in remission. Arch Gen Psychiatry. 2003;60(4):402-407.

19. Reinares M, Colom F, Sánchez-Moreno J, et al. Impact of caregiver group psychoeducation on the course and outcome of bipolar patients in remission: a randomized controlled trial. Bipolar Disord. 2008; 10(4):511-519.

20. Keck PE Jr, Perlis RH, Otto MW, et al. The Expert Consensus Guideline Series: treatment of bipolar disorder 2004. Postgrad Med Spec Rep. 2004: 1- 120 